\title{
Study on the Commercial Photography of the Post Photography Era
}

\author{
Daewook Kim ${ }^{1}$ (First Author) and Joungwoo Joo ${ }^{2}$ (Corresponding Author) \\ ${ }^{1}$ Faculty of Photography and Film, Daegu art University, South Korea \\ ${ }^{2}$ Faculty of Multimedia, Digital imaging major, Kyungwoon University, South \\ Korea \\ E-mail:real7610@daum.net,jw4150@gmail.com
}

\begin{abstract}
The beginnings of digital photography were humble, but now are overwhelming the silver halide photography in 2015. The term Post-Digital Photographic Era mentioned by Fred Ritchin refers to a characteristic image of digital photography with the appearance of digital photography and the beginning that the surrounding infrastructure changes. It means the era that the value framework that the existing photography had changes and a new paradigm begins to appear. Such a new paradigm is giving direct changes, i.e. technology generalization, to photographers and predicting drastic changes to society, culture, politics, and economy. This study looks at the present of photography through literature review of analog photos and digital photos from 1839 to 2015 and based on this literature, investigates the currently-emerging trends of photography and the postphotographic era. It also looks at various cases of technological advancement and presents the future directions for photography. Roland Barthes thought that photography is what was passed away, what was in the past, and what was dead. Through consideration on digital photography, virtual reality, and 3D image - products of various efforts for reviving such dead photos, it looks at the world of visual illusion, ultimate purpose of something virtual and studies the images of photography beyond being ubiquitous, the end of desire for a graphic virtual world.
\end{abstract}

Keywords: Digital photography, 3D image, Post-Digital Photographic Era, Virtual world, Ubiquitous, Commercial photography

\section{Introduction}

\subsection{Purpose}

We are now living in the post-photographic society [1-3]. The development of technology is giving changes to the discourse on photos. The concept of photography is expanding to the digital photography and to stereoscopic image with the scientific changes. The expansion of such a concept is making cultural and social changes. The studies on such a transforming photographic era steadily continue, but the range of such a study is relatively narrow. Accordingly this study diagnoses the flow of analog photo and digital photo and considers a new impact in line with the movement to the postphotographic society, through about 150 years of literature records ranging from analog photos to digital photos. It also looks at the future digital photography by studying on the discourse of digital photography regarding reproduction, extension, and transmission of digital photography depending on digitalization and prospects on the post-photographic era. This study aims to understand the paradigm of post-photographic era by summarizing up the existing discussion, especially on the changes in the image of photographer in the 
post-photographic era (power shift), changes in camera(development of technology), and social changes (cultural change).

\subsection{Methods and Body}

\begin{tabular}{|ccc|}
\hline Methods & & \\
1. Literature review & 2. Focus group interview & 3. Content analysis \\
\hline Theoretical phase & Field work phase & Analytic phase \\
\hline
\end{tabular}

To perform this study, this study conducts a literature review by collecting and analyzing various descriptive data related to the subjects of this study. This study will succeed and associate with the previous studies regarding related contents by collecting, selecting, classifying, analyzing, and reviewing the previous studies. This study considers the previous studies by conducting literature review with such keywords as postphotography, digital photography, hyper-photography, and visual characteristic.

\section{Literature \\ Review \\ Procedure}

Literature collection $\rightarrow$ Data analysis $\rightarrow$ Content-specific selection and classification $\rightarrow$ descriptive data analysis $\rightarrow$ technology $\rightarrow$ review

\section{Body}

\subsection{Developmental Directions of Digital Technology}

Why does it change from analog to digital? Every invention has a reason, and has a mission as well. The development of digital technology can be granting us a certain sense of mission. This study aims to identify and predict which way is given to us. We usually understand digital photography as silver halide photography. This is similar to a speechless carriage as mentioned by Fred Ritchin [3]. When a car appeared first, people recognized the car as speechless carriage. This means that they understood the car as a local part by linking the roles and changes of a car to those of the existing one. Digital photography is also seen from the category of the existing photos, like the metaphor of speechless carriage. However, such a seeing frequently left us assignments such as technology generalization and cultural gap, thus making it difficult for us to understand the digital photography. So we need to see it from a different perspective. It is necessary to understand the digital photography as an extension of the existing photo, but the appearance of a new tool. Digital photography is like a pencil to modern people. Every person takes a photograph, like everyone can make something with some hand movements, like everyone uses a pencil from moment to moment, and like everyone types in a computer. The time has come when hundreds of millions of people can use photos. But, with the phenomenon of technology generalization, the number of people who will pay for the cost of pressing the shutter declines. Such a phenomenon seems to be accelerated. The pursuit direction for photography was vivid. The increase in the number of picture elements in digital camera made photos more realistic and 3D photography made photos and our reality more difficult to be distinguished. The gradual development of camera will continue until the photography can become reality as mentioned above. Furthermore, the development of digital photography is transforming itself from the 
reproduction of material image(s) to non-materialistic world. This is to pass the limit on the reproduction of material world. Now the pixels consisted of bits exist simultaneously through web. The material photo now begins to act as electronic non-material.

\subsection{Characteristics of Digital Photography}

In photography there's myself reflected in the mirror, an image reflected, and a person who watches it. Through my reflection, the truth that I cannot deny about my existence is made. The image reflected in the mirror seems real, but is not real. There is a possibility that it was real, but there is no possibility that it was not real. So is the memory. Like the person who did not see an apple cannot remember any apple, the photographer who did not see an apple cannot take a picture of an apple. However, the advent of digital photography goes beyond the memory or the image met in the mirror. Such signals as 0 and 1 can produce the world that could not be seen through the digital compositing relatively easily [4]. The representative discussion, among various discussions on the analog photography, is mirror and window. There are two concepts on the photography: one view that photo is the mirror that one can see oneself and another view that photo is the window that one can see the world. Photo resembles me because it is selected through my thought. Also, it is a window because I can see the world through it. So photo sometimes becomes a mirror, and other times, becomes a window. In other words, there are various layers of photo ranging from the most distinct image of mine to the most visible outside world. The discussion on the mirror and window can also be seen as the discussion on how to take a photograph by a photographer. Or it can also be understood as a discussion on whether the object to see should be me or outside world. With the appearance of digital photography, photo takes on more complex patterns. With the appearance of digital photography, reinforcement of web, and reinforcement of search tool, photos are now searched and classified immediately. This means that beyond mirror and window, the world of digital photo image glitters like mirror balls. If the light of 'search' is released, the portion into which the light is given glitters and comes out. If the mirror and window is the discussion on the two-dimensional plane, the mirror ball glitters in hundred or thousand directions. If one sheet of photo was important in the past, now the moment when dozens of photos are seen may become important. This enables an immediate look at the photos from dozen and hundred dimensions. These dimensions indicate seeing thoughts and views of various people at one time through the light of 'search.' Such thoughts and views are mixed and combined like mirror balls. In the twodimensional planar discussion, if understanding one sheet of meaning was important, in the situation of mirror ball, color, harmony, etc may become more important. This is consistent with the fact that expert photographer's discussion on the high-dimensional value of photos is relatively decreasing. Images should be seen not only gradually, one by one, through the display of the collection of works but also through the phenomenon brought about by the expression of collective images such as tumbler, pinterest, Flicker, and Google image search. The expression of collective image calls us to select photos selectively. In other words, if the existing photo was one-dimensional, now the numerous images thrown by a photographer are used by a reader in the manner of selecting them in a selective way. This will finally induce a visual competition of images. Or numerous images must be arranged by someone. In other words, it is expected that a more specific adjustment will appear regarding the image search.

\subsection{The Present of Digital Photography}

Prof. Shoukhrat Mitalipov in the college of health sciences, University of Oregon in the U.S. succeeded in human stem cell cloning in 2013. This means that we will be able to begin to copy a body part using the stem cell within a few years and human cloning will be easier like taking a photograph in ten years. Currently, in case of digital photography, you can give various changes to the photo easily like genetic modification. Digital 
photography currently can change skin tone, eye color, a volume of eyebrow, hair, and body form with a Photoshop. I, in the photo, am faced off into an image different from me. I am changed in various ways under the pretext that doing so is more beautiful. The manipulation of such pixels is much easier in digital photography as well as in portrait photography. This simplicity of manipulation is giving the public a belief that photo is not true. If so, how can we change and reinforce photos? We can change ourselves and transforming the real world into the world viewed by an author is possible. Photoshop can be seen as a program that can be used to reinforce photos and change them. Here, reinforcing refers to enhancement of color, tone, atmosphere, etc into the directions as desired. In case of change, it usually refers to compositing. Compositing indicates combining various things. So you can make and add what was not existed before or subtract what was existed before. In case of reinforcement, it is difficult to say that reality has been changed. But in case of compositing, it is regarded that reality has been changed. Various discussions on to what extent compositing should be allowed, of course, have been developed in photojournalist ethics reinforcement, etc, but there are yet few specific standards on this. If a news photo has a mission of delivering objective facts, such a discussion is so important. Photo has an aspect of truth and aspect of falsity, which is a modified form, like Jasus's face. This is also applicable to the digital photography.

Walter Benjamin ordered that looking at how art was transformed must be preceded raising a question if photo is art. Digital photography also consists of pixel and this boils down to signal $[5,6]$. With the material of 'signal,' it is expressed in various ways: advertisement photo that stimulates human's desire, news photo that tends to be a report, pure photo that spreads one's own thoughts, taking a photo as an entertaining element for fun and interest, photo as evidence, photo as observation, etc. All we have to do now is to look at how much we can extend the role from the existing photo through digital photography.

\begin{tabular}{l|l|l}
\hline & Role as the existing photo & \\
\hline 1 & Role as presenting social issues & News photo, documentary photo \\
\hline 2 & Role as art & Artistic photograph \\
\hline 3 & Role as pursuit of desire & Advertisement photos, pornographic picture \\
\hline 4 & Role as various data & Photo material, scientific proof \\
\hline 5 & Role as attractions & Ordinary people's photography shooting \\
\hline & $\begin{array}{l}\text { Role with the appearance of digital } \\
\text { photography }\end{array}$ & \\
\hline
\end{tabular}

Photography as Purpose with Evidence - Is it really possible to trust on the value of photography as evidence? In general, the phenomenon in the photo(s) is a scene of facts although it might have been distorted except that there is a realistic problem in that it is easily manipulated [7]. For this to be applied as evidence, objectivity should be maintained as much as possible. The world within the frame tends to be a new place. So it is difficult for truth to be in here. However, it is obvious that indeed photos are fragmented, but only truth exists in them.

Photography as Emotional Purpose- There are various emotional purposes such as my perspective, pleasure for me, pleasure for others, pleasure for showing off, etc. Photo is a tool like a time machine that can connect the memories in the past with those in reality. Looking at the photos shot in the past enables recalling of the old times. Photo shows a fragmented space, looks at an object from a fragmented perspective, and can contain sound, smell, taste, and feeling. Nevertheless, it cannot become a perfect past because it cannot return to the past perfectly. However, it is possible to dwell on what was not felt then one by one. This is because photo can make up for what could not be handled by a memory. Memory is easily distorted and forgotten, but the scene in the photo records it. 
One knows that this is definitely false, but falls into the moment unwittingly. One is possessed by a spirit, believing that falsity is truth.

Photography as Interest- It is an interest in a new medium. If a new ride appears, can you still enjoy photos? In taking a photograph, there is a certain shooting desire. This is why the sound of shutter is necessary. It is the photography as pursuit for desire enjoying the photos taken. The collection of photos that one begins with the purpose of accumulating desires is photography as interest frequently enjoyed by people.

Photography as social issues- The role of the existing photo was in forming social public opinion imposing the value of truth by raising social issues. However, the issue of 'truth of photography' has continued to become a problem and the belief that photos can be manipulated is always being suspected in this era of digital photography. What on earth is photo manipulation and how much photo manipulation should be allowed? In photo, perspective is selected through object selection, frame, focus, etc. This can make various distortions. Those who have different views will have a different look at image production and make prejudiced crop photos on their own terms. Photos can be produced or composited. Image transformation and manipulation always have a certain purpose. It is thought that it would be appropriate if judged according to such a purpose. Filtering is also required, for example, would it be helpful for the common good? Or would it be necessary for an individual's right? In the end, the allowable scope of manipulation should be determined according to the standards of judgment and in case of photo manipulation, the allowable scope should be different according to its purpose. A photojournalist makes Ethics Declaration commonly used even in the era of analog photography and pursues for truth in order to deliver truth. However, despite such emotional efforts among photojournalists, if the control and manipulation of images rather than a photographer is wanted, there might be an intention to control a society by preoccupying the images searched with search. In case of search, if the images wanted by a manipulator are presented first, the public will be swayed by the images. The photo images penetrate into the public easily and the photos tell the public that such photos are true. This is the power of photos. Like this saying, "one sheet of photo is much stronger than a hundred words," various kinds of information can be presented immediately to the entire world in real time as visual stimulation at a truth-like atmosphere. As a numerous number of information in digital photography are changed and adjusted into various forms, many questions have been raised on the truth of photography, but it cannot and should not be neglected that the photography as truth is the most important part of photo. The difference between photo and picture lies only in the truth. Photos are made by lights or electronic signals rather than human hands. Here, subjective, individualistic, and human intervention is impossible. This is the photo. Based on this, photos can obtain truth and temporality. Much information is distributed and delivered through truthful photos and have had its value as information. Such information delivery and hot news should continue even in the digital era where falsification and modulation are easily possible and for this to be accepted as truth, it is necessary to develop standards and evaluation systems. Now when the dispute on "if photo is art or not" has become unnecessary, the dispute on "if digital photo is true or false" is also useless. Digital photo is strictly true as long as it is not given any transformation. It is seen that the discussion on how much the present can be changed is more important rather than the discussion on "if digital photo is true or false".

\subsection{Photographer's Transition of Power in the Post-Photographic Era}

With the invention of films and the development of cameras, photos became experts' market. By declaring "Kodak, you take a shot! The rest is on us," photos became popular, but here, there is a strict difference between expert and layman and the general public's envy of experts is still the same. Photos are taught in universities and classified into two markets: expert and layman. To look at the phenomenon brought about by the appearance 
of digital photography, the initial digital market was the period when expert's interests were amplified. At this time, taking a digital photo itself was not easy and the image quality was not good. However, with the maturity of technology, digital technology became easy and amateur's catch up with expert phenomenon is accelerating. This can be explained by the advanced equipment user inversion phenomenon: amateurs possess more advanced equipments than experts. Digital bag, for example, pursues for high definition with high-priced equipment. In this high-priced market, amateurs who enjoy their hobbies are purchasing more than experts. Another aspect is in post-production. Digital photos can be produced easily [8]. And the produced photos can be reproduced in various forms. Retouching photos can adjust and reinforce tone or color or compositing or changing shapes is possible. In other words, photos can be changed by this post-production and the scope has also expanded. Accordingly, all people as well as photographers have the right to do post-production and the reduction in the scope controlled by a photographer has finally transferred power to the public. Moreover, with such a weakened power, the right to editing became more important than the photo's intrinsic value.

\subsection{The Point that Digital Photography Sees- Seeing Beyond What's Seen.}

If a photo consists of pixels, is it photo or a mass of pixels? In other words, depending on the aspect of recognition, photos have a nature that they are recognized as photos. In case of film, we thought silver halide particles as object. What we regard as photos is in the end what is seen beyond the properties of an object although it is seen through photos. In other words, it is looking at the world consisted of points like halftoning in a newspaper and recognizing it as real images. In other words, it is accepted differently depending on how the image(s) of particles are constructed. If so, pictures can be seen to be expressed in various ways through the particles of paints. However, brushes and paints made an illusion of such a true nature, but lacked in causing an illusion. Artists developed perspective, coloring, and shading as methods to cause an illusion. However, a picture still remains as a picture. To look at a paper cup, it is made with paper and such a paper is made with wood. If shape and color are given to this paper, we recognize it as a cup and at the moment, the cup forms its own cup beyond the form of paper or wood. Let's return to the pixel again. Digital photos consist of pixels, but if the pixels gather together and have colors, at the moment, the pixels are recognized as a certain image and after it's recognized so, it constitutes its shape as an image. This is digital photography. In the end, we look at the things beyond while looking at points. With the appearance of digital photography, there is an increasing interest in the hidden side of photos rather than photos seen as evidence. This suggests that there is an increasing interest in non-materialistic eyes that see beyond what is seen.

\subsection{Changes with the Difference in Shooting Method}

What was shown by Muybridge through photos was that a horse runs with four feet. People in the past could not see a horse running exactly. In fact, photos made the photos that could expand things with the development of microscope and with the development of telescope, we are seeing the universe. Sounds and waves are seen by converting them into water or other media or air current and electromagnetism are seen by using the high speed lighting. Through this, we are exploring and seeing what our sight does not reach in various forms. In relation to the existing photos, various methods to record what we know and what are physically existent are being studied and recorded. The researches on recording non-materialistic existence are possible. Photos have concentrated on the visible world so far [9]. In modern times, however, the visual, materialistic world is moving to the non-visual, non-materialistic world. The studies on photos can now be conducted with efforts on how the non-visual world rather than the visual world can be visualized. This can be understood as exploration of the non-visual area or as ambiguous emotional line represented by aura, punctum, and atmosphere. If any device to record 
other dimensions through non-visual area or other methods is developed, another discourse from another perspective will be presented. Photos implement a perfect fact that was not presented in the existing picture in two-dimensional way. This granted a status that was not owned by the picture of reality into photos. This is why the photos in the post-digital era need to be explored regarding a new visual area. As we are too accustomed to the visual discourse made by Daguerre and Talbot who invented silver halide photography, we might have thought it everything like the fish in the water [10, $11]$.

\section{Conclusion}

With the popularization of camera, the production and consumption of image(s) is increasing geometrically. There are various things such as cellular phone, web camera in a notebook, DSLR, and scanner. The act of taking and possessing photo records, specifically records important points of site. If analyzing the meta data of such photos, what humans intend to record, what they intend to possess, and what they prefer can be identified. Photos can be transmitted or stored in real time, especially regarding various information such as location and shooting information as well as one's own records, through a surveillance camera and a video camera as well as a digital camera. Such information are uploaded into web, linked, searched, and restored. This means that we are in the era of image explosion. In addition, images are stored and searched with one keyword. In the past, letter-centered search was dominant, but currently, video and imagecentered search is increasing in its proportion. The conversion from the illiterate era to the dummy era, being literate is communication, and any argument is possible. If the public are literate, it is the way to democratization. In other words, power is disintegrated. With the explosion of various ideas, the increase of various thoughts, and the appearance of various rookies, the existing authority collapses and writing becomes ones mean. In other words, learning to be literate is learning how to write. The development of Photoshop and camera should be understood as a visual stimulation image rather than understanding photos as photos. Photos become a new expression mode and writing. The camera technology and sight-based technologies are developing into the technology on optic nerve. The stimulation on optic nerve will make the perspective more duplicated from the reality difficult to be distinguished. Now photos will develop into a three-dimensional image. The end of such a three-dimensional image is also predicted because the threedimensional image will finally be a virtual image. Such a virtual image world will change into the age of reproduction through genes, or the age of optic nerve using brain science. Photos will transform itself from the imaging technology to the age of genes or recognition. As seen earlier, the changes and the width of the changes of digital photography have now become large enough to detect such changes. Now, from such changes, a revolutionary leap must happen in the post-photographic era.

We usually understand that digital photography has been developed from the photos in the film era. This is similar to a speechless carriage, as mentioned by Fred Ritchin. When a car appeared first, people recognized car as speechless carriage. This is an understanding of local parts only by linking the role and changes of a car to those in the existing era. Digital photography is also seen from the category of the existing photos like the metaphor of speechless carriage. However, such a seeing frequently left us with such assignments as technology generalization and cultural gap and made us difficult to understand the digital photography. So we need to look at it from a different perspective. It needs to be understood as appearance of a new tool rather than an extension of the existing photos. The camera will gradually develop until photos can be real rather than virtual. Anchoring of image, optical idea, appearance of machinery that can copy the world through these as mentioned right before, and now we can copy the world through three-dimensional video image as well as planar image. Anyone can copy the world easily. Such a reality foretells the end of scientific debate over photos. For photos or 
mechanical and scientific properties to be magnified again, heart should be recorded or stored or what could not be seen before should be seen as if showing a spirit. We are entering the era when it is difficult to feel the fantastic aura in the past from the digital photos. Photos are just produced and consumed. For photos or mechanical and scientific properties to be magnified again, heart should be recorded or stored or what could not be seen before should be seen as if showing a spirit.

\section{Reference}

[1] J. C. Choi, "Photography's New Turn in the Post-Photographic Era," Council for advanced media \& Moving pictures, vol. 13, no. 4, (2014), pp. 85-113.

[2] D. W. Kim, "Study on the changes of photography through the post photography era", Advanced Science and Technology Letters Vol.101 (Art, Culture, Game, Graphics, Broadcasting and Digital Contents, (2015), pp. 1-4.

[3] F. Ritchin, “After photography”, W. W. Norton, New York, (2009).

[4] L. Yong-Hwan, "New Photographic paradigm on Digital Age", The Journal of Korean Documentary Photography Society, vol. 3, (2002).

[5] L. Kyoung-Ryul, "The paradoxes of Digital Photographic Image and the Aura," Center for Cross Culture Studies, vol. 12, no. 2, (2008), pp. 299-323.

[6] R. Barthes and S. Sontag, “About Photography,” Hyundai-Mihack-sa, Korea, (1994).

[7] W.-R. Kim, "Photography and text: From invention of photography to digital photography", Noon bit, Korea, (2006).

[8] M. Lister, The photographic image in digital culture, (1995).

[9] W. H. F. Talbot, "Introductory Remarks" in The Pencil of Nature, Longman: London, (1844).

[10] J. M. William, "The Reconfigured Eye: Visual Truth in the Post-Photographic Era", Digital image, (1992).

[11] J. M. William, "What do pictures want? The lives and loves of images", The University of Chicago Press, Chicago, (2005).

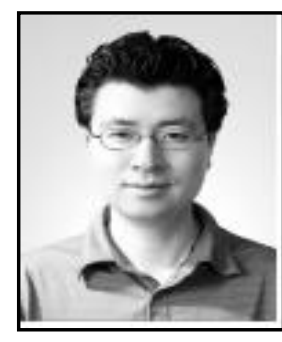

Dae wook, Kim, Ph.D. Chung-Ang University: Korea, Major in Commercial photography (2014)

M.A. Brooks Institute of Photography, Santa Barbara, USA Major in photography \& Related Media (2007)

M.A Chung-Ang University: Korea, Major in Commercial photography (2003)

B.A Chung-Ang University: Korea, Major in Commercial photography (2001) 